\title{
Plasma Injection and Atomic Physics Models For Use In Particle Simulation Codes
}

\author{
Richard J. Procassini \\ Massively Parallel Computing Initiative \\ Lawrence Livermare National Laboratory \\ Livermore, CA 94550 \\ and \\ Elentronics Research Laboratory \\ University of California \\ Berheley, CA gA720
}

UCRL-ID--107219

DE91 015092

12 Јиле 1991

\begin{abstract}
Models of plasma injection (creation) and charged/neutral atomic physics which are suitable for incorporation into particle simulation codes aie described. Both plantar and distributed source injection models are considered. Results obtained from planar injection into a collisionless plasma-sheath region are presented. The atomic physics package sintulates the charge exchange and impact ionjzation interactions which occur between charged

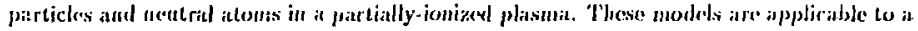
wide range of problems, from plasma processing of materials to transport in the edge region of a tokamak plasma.
\end{abstract}




\section{The Planar And Distributed-Sourse Injection Models}

In order to maintain an equilibrium particle density in a plasma, it is necessary to have a particle source which balances the loss from the system. The method of particle creation or injection that is chosen for a specific system is usually determined by the means of plasina confinement. Gas puffing, frozen-gas pellet and neutral beam injection have been entployed as a means of fueling magnetically confined plasmas. Cold, neutral gas is introduced across the confining magnetic field into the plasma, where it is then ionized via collisions with the plasma particles. In bounded systems, such as dindes and single-ended $\mathbf{Q}$-machines, particles are emitted by one of the bounding plates and are lost via flow to the other plate or perpendicular diffusion to the walls of the device. The dominant "source" of plasma in the scrape-off layer (SOL) of a tokamak is cross-field transport of particles across the separatrix. These particles then flow along the open magnetic field lines to the divertor plate or limiter, where they are absorbed or recycled as neutral atoms. Each of these systenis may be modeled through the use of the planar and distributed source injection packiges in the code DIPSI [1], which are derivatives of the planar source model in Lawson's code PDWl $[2,3]$.

\subsection{Methodology}

Planar and distributed source algorithms are used to iuject particles into a system from the left-hasd boundary and a region of finite spatial extent within the system respectively. The velocities of the injected particles are chosen from it prescribed distribution function. A drift velocity $y_{0 n}$ about which tlie sourec distribution function is centered may be specified for cacle species s. The following subsections describe the methods that are used to determiac the position and velocity of the injected particles.

\subsubsection{The Distributed Source Model}

The distributed source model is used to represent the volunetric production of jon. electron particle pairs. It is therefore applicable for the simulation of neutral-particie injection into magnetically confined plasmas, the ionization of neutral gas or the uransport of plasma across the separatrix of a tokanlak. 
The velocity of each injected particle is chosen from the normalized source distribution functions $S_{\|,,}\left(z, v_{\|}\right)$and $S_{\perp, \Omega}\left(z, v_{\perp}\right)$ for each species $s$, which have the general forms

$$
S_{\|, s}\left(z, v_{\|}\right) d v_{\|}=h(z)\left|\nu_{\|}\right|^{\eta_{s}} \exp \left(\frac{-m_{s} v_{\|}^{2}}{2 k T_{\|, a}}\right) d \nu_{\|}
$$

and

$$
S_{\perp, s}\left(z, v_{\perp}\right) d v_{x} d v_{y}=h(z) \exp \left(\frac{-m_{\perp} v_{\perp}^{2}}{2 k T_{\perp, s}}\right) v_{\perp} d v_{\perp} d \theta
$$

where $h(z)$ is the source intensity profile in the range $[0,1], k$ is Boltzmann's consitiul, and $m_{s}, T_{\|,}$and $T_{\perp,}$, are the mass, parallel and perpendicular source teinperatures for species $s$ respectively. The exponent $\eta_{\text {s }}$ allows one to choose either a Maxwellian $\left(\eta_{s}=0\right)$, $\|_{11 x}$ $\left(\eta_{s}=1\right)$ or more general distribution function for the species $s$. The $v_{\perp}$ that follows the exponential in (2) is present because the perpendicular direction comprises two degrees of freedom (the $\bar{x}$ and $\bar{y}$ directions), such that $d v_{x} d v_{y}=v_{\perp} d v_{\perp} d \theta$, whereas $d v_{2}=d v_{\|}$.

The parallel and perpendicular source velocities are generated from these source distribution functions at the start of the run and are stored in arrays. The perpendicular source velocities are stored in increasing order from $v_{\min }=0$ to $v_{\max }$, while the paral. lel source velocities are stored in base-2 bit-reversed order for "iquiet" injection from the planar source). (See Reference [4], pages 393-394 for a discussion of bit-reversed numbers and their application to particle simulation methods). Each of the species has an arrity for parallel and perpendicular source velocities. Upon injection, the index of the paralle! and perpendicular velocity arrays, and hence the valies of $v_{\|}$and $v_{\perp}$ are chosen randemly for each particle, which tends to minimize correlations of the injected velocity components.

The source region is defined by the center location $Z_{\text {os }}$ and the width $\Delta Z_{S}$. The source intensity function that has been employed in DIPSI is $h(z)=1$, although more general intensity profiles are possible. The particles are injected uniformly over the source region via

$$
z_{i n j}=Z_{o s}+R_{N} \Delta Z_{S}
$$

where $R_{N}$ is a random number in the range $\left.(-0.5,0.5)\right]$. The electrons and ions are injected at the same location. Since the electron and ion velocities are, in general, not the same, it is possible to have cliage separation that will result in an electrostatic source slieatl.. The velecity components of the injected particles are then used to calculate the relativistic factor $\gamma$, the parallel nomentun $p$; and the maguetic moment $\mu$. These quantities are 
then written to the permanent particle arrays. If the number of particles injected per time step exceeds the free space at the end of the pernianent particle ariays, the arrays are dynamically expanded by the input variable $N_{\text {expand. }}$. In order to reduce the amount of tine spent expanding the arrays, $N_{\text {expand }}$ is chosen suclt tlat there are of order 100 time steps between succssive array expansions.

\subsubsection{The Planar Source Model}

The planar source model may be used to simulate the injection of particles by the cathode of a plasma diode or single-ended Q-machine, or aiternatively, may represent the Rux of Maxwellian particles which is crossing a plane boundary. The planar source morlel is similar in many ways to the distributed source package. One major difference is the normalized source distribution function, which for particles emitted from a plane is a flix distribution

$$
S_{\|, s}\left(v_{\|}\right) d v_{\|}=\left|v_{\|}\right| \exp \left(\frac{-m_{s} v_{\| \mid}^{2}}{2 k T_{\|, s}}\right) d v_{\|}
$$

and

$$
S_{\perp, s}\left(v_{\perp}\right) d v_{x} d v_{y}=\exp \left(\frac{-m_{s} v_{\perp}^{2}}{2 k T_{\perp, s}}\right) v_{\perp} d v_{\perp} d \theta
$$

The parallel and perpendicular velocity components of the injected particles are chosen in the following manner. The perpendicular velocity component is cloosen randomly out of the perpendicular-velocity source array. For the parallel velocity component, the code maintains an integer counter. This counter starts at one and increases by onc for each particle (of either species) that is injected. When a counter exceeds the length of the array, the counter is reset to one and then increased by one for each additional particle ili,jected. This counter is then used as the element that is to be chosen for injection from the bitreversed parallel-velocity source array. This method ensures that the velocity components of the injected particles are chosen uniformly from the range $\left[v_{\min }, v_{m a r}\right]$.

The positions of the injected particles are calculated in the following way. Each particle is injerted at the left boundary $(z=0)$, but its position at the end of the injection routine is related to its velocity and when it was injected during the time step. The time step $\Delta /$ is divided by the number of particles of the given species that are in be injected in that time step to give the time between injections as $\tau_{i n j}=\Delta t / N_{i n j}$. The position of the $i-t h$ 
particle at the end of the injection routine is then given by

$$
z_{i}=v_{\|, i}\left(N_{i n j}-i\right) \tau_{i n j}
$$

Finally, the velocity components of the particles are used to calculate the relativistic factor $\gamma$, the parallel momentum $p_{*}$ and the magnetic moment $\mu$, which are then written into the permanent particle arrays. If necessary, the arrays are expanded via the previously described method.

\subsection{Simulation Results}

The planar source injection model is used to simulate a flux of Maxwellian particles which cross a specified plane in space, and proceed to flow to an electrostalically floaling, conducting plate. This absorbing wall is the system boundary on the right side, while the "injection" plane is the left side boundary. It is assumed that there is a collisiona!, dis. eributed source located to the left of the computational domain, where particles of specius $s$ are created and maintained in a Maxwellian velocity distribution of characteristic tem. perature $k T_{s}$. As these particles enter the system across the left system boundary, the electrons are, in general, travelling faster than the ions. As a result, a source shcath is generated which attempts to equilibrate the particle fluxes in the region just to the right of the left system boundary. Particles which exit the system across the left system boundary are reflected, with a velocity chosen from an untruncated half-Miaxwellian of temperature KT, travelling to the right. This boundary condition is included to replicate the collixicnial relaxation processes which take place in the region to the left of the system.

The model assumes that the plasma is untnagnetized and collisiouless (except for the re-thermalization process which takes place at the left system boundary). E(pal uumbers of electrons and jons per time step are "injected" across the source plane $z=0$ with parallel velocity distribution functions that are hadf-Maxwellian travelling to the sight and perpend:cular velocity distributions that are Maxwellian. Hence, the model is 1-D (one spatial coordinate) and $2 . V$ (two velocity space coordinates). For the results prescnted below, the system length $L=2.0 \mathrm{~cm}$ corresponds to approximately $26.6 \lambda_{D e}$ for the equilibrium deusity $n_{e}$ and source temperature $k T_{e}$. The source temperature ratio is $k T_{e} / k T_{i}=1$ and llie mass ratio is $m_{i} / m_{e}=100$. The drift velocity of both the electrons and ions is $v_{0 c}=v_{0 t}=0$. 
The equilibrium phase space scalter plots for the electrons and jons are shown in Figures 1 and 2 respectively. The corresponding potential profile along the $z$ axis is given in Figure 3. Since the system is unmagnetized and collisionless, there are no forces present in the model to change the perpendicular velocity distribution of the particles. 'This is borne out by Figures $1 \mathrm{~b}$ and $2 \mathrm{~b}$, which show that the perpendicular-velocity plase space for cach of the species remajns Maxwellian as the particles traverse the system.

Figure 3 indicates that there is source sheath on the left side and a collector sheath on the right side of the system. Each of these sheaths have formed over four to five electron Debye lengths, which is a typical distance over which deviations from quasineutrality (due to the injection of particles at the left boundary and the net negative charge on the collecior plate) are shielded out in a plasma. The parallel-velocity phase space plot (Figure 1a) shows electrons that apparently form a cut-off or truncated Maxwellian distribution function. This structure is a consequence of the equilibrium potential profile. Only electrons that are energetic enough to climb over the collector sheath potential hill are absorbed on the plate. The remaining electrons are reflected by the collector sheath. Since the electron distribution function to the left of the collector sheath region is essentially a right-going lialf-Maxweilian (the electron distribution function is modified by the source sheath to some extent), the refected electrons form a left-going, truncated half-Maxwellian distribution function. The velocity cut-oif is duc to the electrons that were energetic enongh to traverse the collector sheath and impinge upon the absorbing plate. The ion parallel-velocity phase space plot (Figure 2a) shows that the ions are accelerated towards the plate by the monotonically decreasing potential. Therefore, all of the ions that are injected across the plane at $z=0$ will reach the absorbing plate. The number of electrons that reach the plate is such that the electron and ion currents at that locitton atre edual, which is recessary to maintain a steady-state plate charge and coltector sheath drop.

The distributed source madel has been used in to study the effect of the source distribution function $S(z, v)$ on the width and magnitude of the collisionless source and collector sheaths [5], to investigate the effect of Coulomb collisions on the potential structure and plisma transport in the plasma-shesth region $[6]$, and to stusly the effect of charge-exchange and ionization of recycled neutral particles on the presheath potential variation in the SOL of a hight-recycling diverted tokinmak [7]. 


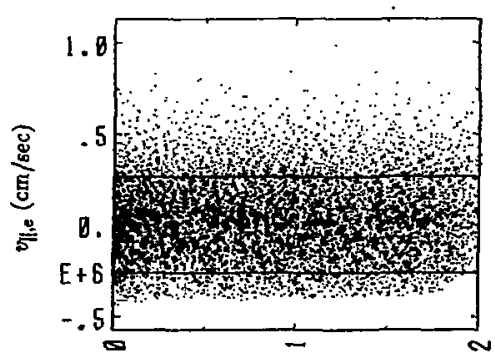

Axial Position (cm)

(a) Electron parallel-velocity phase space scatter plot.

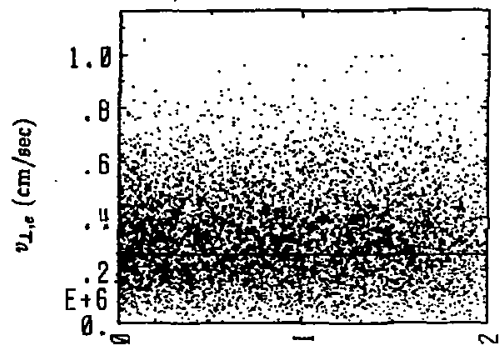

Axial Position (cm)

(b) Electron perpendicular-velocity phase space scatrer plot.

Figure 1: Electron (a) parallel-velocity and (b) perpendicular-velocity phase space scatter plots for the case of Maxwellian particle injection into the plasma-sheath region adjacent to a fioating, absorbing conductor. The gpecies mass and source teraperature ratios are $m_{i} / m_{e}=100$ and $k T_{e} / k T_{i}=1$. 


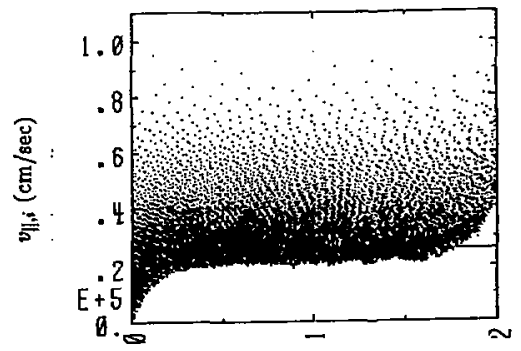

Axial Position $(\mathrm{cm})$

(a) Ion parillel-velocity phase space scatter plot.

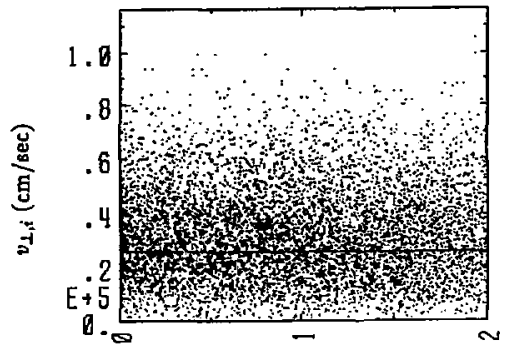

\section{Axial Position (cm)}

(b) Ion perpendicular-velocity phase space scatter plot.

Figure 2: Ion (a) parallel-velocity and (b) perpendicular-velocity phase space scatter plots for the case of Maxwellian particle injection into the plasma sheath region adjacent to a floating, absorbing conductor. The species mass and source temperature ratios are $m_{i} / m_{e}=100$ and $k T_{c} / k T_{i}=1$. 


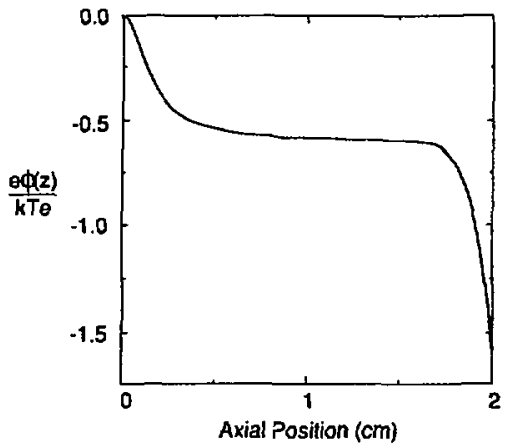

Figuse 3: Potential profile for the case of Maxwellian particle injection into the plasinasheath region adjacent to a floating, absorbing conductor. The species mass and source temperature ratios are $m_{i} / m_{e}=100$ and $k T_{c} / k T_{i}=1$.

\section{The Atomic Physics Models}

The heat load on and plasma-induced sputtering of the surface of a divertor piate in a tokamak is strongly dependent upon the amount of energy that is transferred from the incoming charged particles to the neutral atoms which are located in the vicinity the plate [8]. These cold neutral particles are formed by reflection or "recycling" of the plasma ions from ihe divertor plate, which supplies the clectrons which are required to neutralize the incident charged particles. The plasma particles undergo charged-neutral collisions with the background gas. These collisions can be either elastic (the combinsid kinetic energy of the particles remains constant, but the trajectories are changed), inelastic (the charged particle frovides energy to place the bound electrons of the neutral particle in all e':rited state) or ionizing (where the energy transferred in the collision is large snough to remove the orbitid electron from the neutral particle) in nature. In addition, a warm plasma ion may exchange an electron with a cold neutral particle, producing a cold ion and in warm neutral atom. In an attenpt to model some of these atomic physics processes, all itupact ioniatiou and 
charge exchange package has been incorporated into the code DIPSI [1].

\subsection{Methodology}

The atomic physics package in the code DIl'SI is used to simulate the effects of cliarged particles interacting with a background neutral gas. The atomic physics models that are incorporated into DIPSI are similar to those in the "PD" series of bounded particle codes from the University of California at Berkeley [12,13], which are used to model the lowionization plasmas employed in semiconductor processing. The neutral gas in a tokamak SOL is produced as plasma interacts with the divertor plate. As plasma ions impact a matcrial surface, they undergo both elastic collisions with the nuclei, and inelastic collisions with the electrons of the plate atoms, leading to a reduction in their kinctic energy. Collisions with the atomic nuclei also lead to changes the direction of the flight path. A fraction of these jons will undergo sufficient pitch-angle scattering to exit from the plate and reenter the plasma. These reflected or recycled particles may have either positive, neutral or negative charge states (having obtained electrons from the plate material). Tlie [raction of these "prompt" reflected particles, their average reflected energy and energy distribution. and their charge state depend upon the energy of the incident ions, the angle of incidence and the atomic charges and masses of the incident and target particles [0]. The plasma ions that come to rest (thermalize) in the atomic lattice of the plate material are another source of neutral particles. These monatonic and/or molecular particles will diffuse lowards the surface of the plate, where they may be relcased by physical (photon or plasma impact) or chemical desorption processes $[10,11]$.

The code DIPSI treats the neutral particles in an ad hoc manner by assuniug that the neutral density decays exponentially with increasing distance from the divertor plate. This sensity profile is obtained if one represents the divertor plate as a planir source of reflected neitrat particle flux. The neutral density e-folding length $\mathcal{L}_{n}$ is taken to be the average ionization mean-free-path length $\left\langle\lambda_{\text {Ion }}\right\rangle$, surch that

$$
n_{n}(z)=\hat{n}_{n} \exp \left(-[L-z] / \mathcal{L}_{n}\right)
$$

where $L$ is the location of the divertor plate (system length), and $\hat{n}_{n}$ is the peak neutral density adjacent to the divertor plate. The average ionization mean-free pall $\left\langle\left\langle\lambda_{\text {ian }}\right\rangle\right)$ and 
the peak neutral density $\left(\hat{n}_{n}\right)$ are treated as input parameters in this model.

The peak neutral density is adjusted in time so as to maintain a constant jonization source term $S_{\text {ion. }}$. At the start of a silnulation, the initial source term is calculated via

$$
\begin{aligned}
S_{\text {ion }}^{0} & \equiv \int_{z^{\prime=1}}^{L} n_{e}\left(z^{\prime}\right) n_{n}\left(z^{\prime}\right)\left\langle\sigma_{i o n}\left(E_{e}\right) v_{e}\right\rangle d z^{\prime} \\
& \simeq \sum_{j=j_{\text {mun }}}^{N G+1} n_{e, j} n_{n, j} \sigma_{i o n}\left(E_{c, j}\right) v_{e, j}
\end{aligned}
$$

where $j$ is the grid point index, $n_{e}(z)$ is the electron density profile, $\sigma_{\text {ion }}$ is the clectronimpact ionization cross section, $E_{c}$ is the local electron kinetic energy and $v_{e}$ is the locil electron velocity. This source term is reevaluated every $N T_{\text {nd }}$ time sieps (where $N T_{\text {nd }}$ is ant input parameter), using quantities that have been averaged over the previous $N I_{n d}$ tine steps

$$
S_{\text {ion }}^{T}=\sum_{j=j_{\min }}^{N G+1} \bar{n}_{e, j} \bar{n}_{n, j} \sigma_{\text {ion }}\left(\bar{E}_{e, j}\right) \bar{v}_{e, j}
$$

where $\mathcal{T}$ is an integer multiple of $N T_{n d}$. The peak neutral density is then adjusted via

$$
\hat{n}_{n}^{T}=\hat{n}_{n}^{0}\left(\frac{S_{i \text { in }}^{0}}{S_{i o n}^{T}}\right)
$$

where $\hat{n}_{n}^{0}$ is initial pcak neutral density that is inpui by the user.

Many of the physical aspects of neutral particle recycling from a divertor plate are included in this constant-ionization model. The flux of neutral particles reflected from tlit plate increases with the flux of plasma incident upon the plate. The flow of warm plasma to the plate is inhibited by the charged/neutral inceractions which occur in che region adjacent to the plate. As the fraction of plasma ions recycled from the plate increases, ihe neutral density and charged/neutral interaction rates will also increase, thereby reducing the recycled ncutral flux and neutral narticle density. In the proposed model, as the plasina density adjacent to the plate increases (as a result of impact ionization events), the neutral density is decreased accordingly. If the plasma density in this region decreases, the neutral density is increased. Note that the model also includes a dependence on the erergy of the plasma in this region through the jonization reaction rate $\sigma_{i s n}\left(E_{\varepsilon, j}\right) \bar{v}_{c, j}$.

The time history of the peal "cutral density during the simulation of traysport in a highrecycling divertor SOL is showıi in Figure 4, Warm plasma ( $k T_{c} \simeq 100 \mathrm{eV}$ ) Aows collisionally towards the plate, undergoing interactions with cold neutral particles $\left(k T_{n}=5 \mathrm{eV}\right)$. Thie 


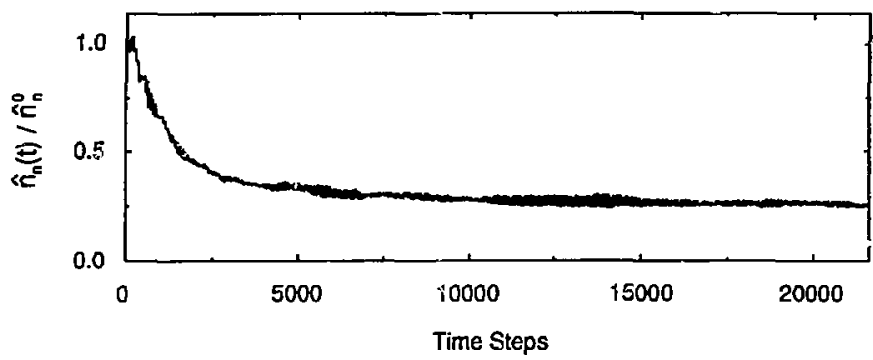

Figure 4: The time history of the peak neutral density in a high-recycling divertor scrape-of layer in a tokamak. The peak density is adjusted every $N T_{n d}=25$ time steps in order to maintain a constant ionization rate.

peak density was adjusted every $N T_{n d}=25$ time steps. The peak density decreases over the first 5000 time steps of the simulation as the plasma density adjacent to the plate increas: due to (i) the influx of warm plasma from upstream and (ii) the ionization of cold neutral particles. The neitral density appronches a steady-state value at approximately the half-way point in the simulation. The high-frequency fluctuations occur on the $N T_{\text {s,d }}$ time scale.

\subsubsection{The Impact Ionization Model}

Neutral atoms may be ionized via collisions with the eharged particles of the plasma. If the charged particles strike the atoms vith sufficient force, one or more electrons will be stripped from the neutral atom. For atoms which ars isotopically identical to the plasma ions, this grocess results in an increase in the plasina electron and ion density.

Since the electron/neutral coltision model it the code DIPSI is intended to simulate plasma recycling from a plasna-facing componert, such as a divertor plate or limiter, only ionization events arising from electron/neutral inter zctions are considered. Elastic- 
scaltering and excitation collisions between electrons and fusion-felevant neutral alous (liydrogen, deuterium, tritium and heliun) are neglected, as are ion-inpact-ionization cvents, since the cross sections for these processes are much smaller than that for electron-impact iorization [14]. This model borrows from the previous efforts of 1. J. Morey and M. Surendra at the University of California at Berkeley [12].

Since the model does not include excitation collisional processes, it is not entirely ap cable for tite simulation of impurity particle (carbon, oxygen, iron, ttickel, etc.) transport. The emission of line radjation by excited orbital electrons (as they make a trausition to a lowes energy state), and bremsstrahlung by free elect rons (as they are accelerated by plasmit ions) are major energy loss mechanisms in a low to moderate temperature SOL plasma. The power emitted in the form of line radiation increases with the number of available orlyitalelectoon energy states, whicl in turn rises with the atomic number of the particle, and the bremsstrahlung power emission increases as the square of the effective atomic number of the plasma. Therefore, even small quantities of inpurity particles can lead to a siguficaut amount of energy loss via radiative processes. Models for these radiative processes should be added to the present atonic pliysics package if one wislies to simulate impurity particle transport.

The probability of the $k-$ th plasma electron having an ionizing collision with a newtral particle during a given time step is a function of the local neutral density $n_{n}(z)$ (defined on the grid points), the kinetic energy (and hence tle velocity of the electron) $E_{e} \equiv \frac{1}{2} m_{e}\left(v_{\| j}^{2}+\right.$ $v_{\perp_{e}}^{2}$ ) and the length of the time step $\Delta l$

$$
P_{i o r, k}=\left[1-\exp \left(-n_{n}(z) \sigma_{i o n}\left(E_{\mathrm{e}, k}\right) v_{e, k} \Delta t\right)\right]
$$

where $k$ is the electron index. The probability $P_{i o n, k}$ is calculated for each electron in a given grid cell. An ionizing collision occurs if a random number $R_{N}$ (chosen from the range $(0,1))$ is less than $P_{i o n, \dot{r}}$. (If the argument of the exponential in (12) is $\ll 1$, such that $P_{\text {ion, } k} \ll 1$, it is possibie to sample every $N_{s k i p}$ electrons, and multiply the right side of (12) by $N_{a k i p}$ to provide a reasonable ionization probability per saripied electron per time step.) 
The electron-impact ionization cross section has the following energy dependence

$$
\sigma_{i o n}\left(E_{e}\right)= \begin{cases}0 & , E_{e}<E_{t h} \\ \hat{\sigma}_{i o n}\left(\frac{E_{e}-E_{t h}}{E_{p l, m i n}-E_{t h}}\right) & , E_{t h} \leq E_{e}<E_{p l, \text { min }} \\ \hat{\sigma}_{i o n} & , E_{p l, \text { min }} \leq E_{e}<E_{p l, \text { max }} \\ \hat{\sigma}_{i o n}\left(\frac{E_{p l, \text { max }}}{\ln \left(E_{p l, \text { max }}\right)}\right)\left(\frac{\ln \left(E_{e}\right)}{E_{e}}\right) & , E_{e} \geq E_{p l, \text { max }}\end{cases}
$$

where $\hat{\sigma}_{i o n}$ is the peak ionization cross section, $E_{t h}$ is the minimum or threshold kinetic energy required to ionize a neutral particle, and $E_{p l, \min }$ and $E_{p l, \max }$ are the limits of kinetic energy for which the cross section las a constant, peak (piateau) value. All of these quantitics are input parameters in the present model. The energy dependence of the jonization cross section for electrons colliding with monatomic hydrogen $\left({ }_{1} \mathrm{H}^{2}\right)$ is shown in Figure 5. Figure 5a showz experimentally measured values [15], wlile Figure 5b shows the values obtained from (13) for $\hat{\sigma}_{\text {ion }}=7.15 \times 10^{-17} \mathrm{~cm}^{2}, E_{t h}=13.6 \mathrm{eV}, E_{\text {pl,min }}=40.0 \mathrm{eV}$ and $E_{p l, \max }=80.0 \mathrm{eV}$. The simple model described by (13) provides reasol a.ble agreenent with measured values over the energy range $0.1 \mathrm{eV} \lesssim E \lessgtr 1.0 \mathrm{keV}$.

Once it has been determined that a given electron will ionize a neutral particle, the encrgy available for the scattered electron $\left(E_{\mathrm{e} 1}\right)$, and the new electron $\left(E_{\mathrm{e} 2}\right)$ and ion $\left(E_{i}\right)$ pair are obtained via an seergy balance

$$
\Delta E=E_{\mathrm{c} 1}+E_{\mathrm{e} 2}+E_{i}=E_{\mathrm{c}}+E_{n}-E_{t h}
$$

where the energy of the neutral particle $E_{n}$ is chosen from an isotropic Maxwelliau distribution of temperature f: $_{n}$ via the relation

$$
E_{\mathrm{n}}=k T_{\mathbf{n}} \ln \left(\frac{1+\delta}{1+\delta-R_{N}}\right)
$$

where $\delta=10^{-8}$ and $R_{N}$ is a randon number in the range $(0,1)$. The argument of the logarithm ranges in value from 1 to $10^{-8}$, sepresnnting the limits on the value of the normialized distribution function $\hat{f}(v)$.

The energy of the scattered electron $E_{e 1}$ is the calculated via the energy-differential cross section [16]

$$
\sigma_{\text {ion }}\left(E_{c}, E_{t 1}\right)=\frac{\alpha\left(E_{e}\right)}{E_{e 1}^{2}+\Gamma^{2}}
$$




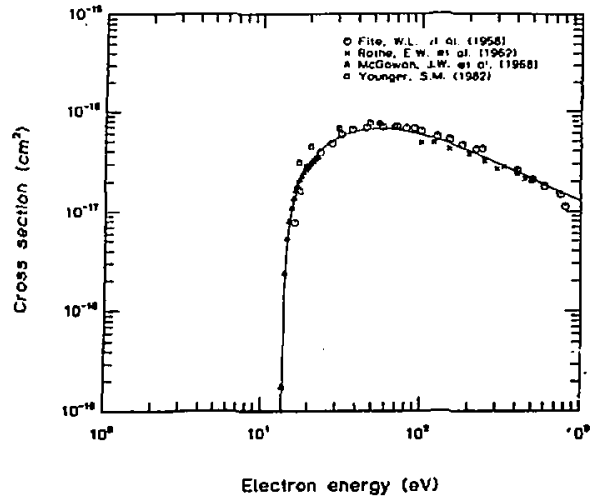

(a) Experimentally measured ionization cross section [11].

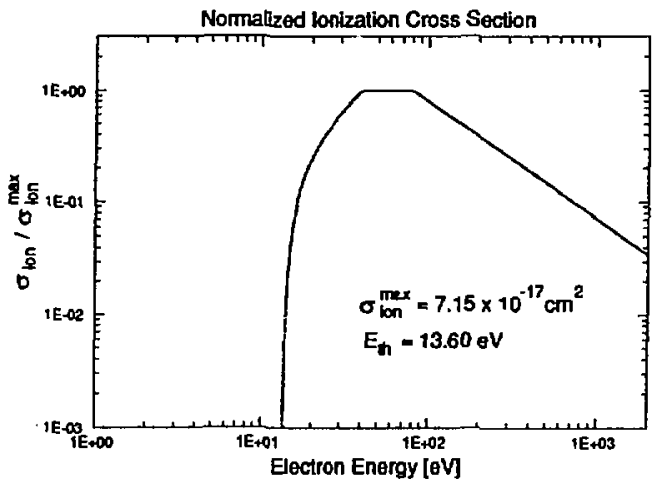

(b) Ionization cross section calculated by the simple model (4.13).

Figure 5: Ionization cross section as a function of energy for collisions between electrons (e) and monatomic hydrogen $\left({ }_{1} H^{1}\right)$ from (a) experimental measurements [11] and (b) from the simple model (4.13). 
where $\Gamma$ is the energy-level width of the cross section. Since the maximum energy available for the scattered electron is $E_{e l, \pi i a x}=\left(E_{e}+E_{n}-E_{t h}\right) / 2$, it is possible to integrate the encrgy-differential cross section as $[12,13]$

$$
\sigma_{i o n}\left(E_{c}\right)=\int_{0}^{E_{e j, r n a x}} \sigma_{i o n}\left(E_{e}, E_{c 1}\right) d E_{\mathrm{c} 1}
$$

such that one obtains

$$
\alpha\left(E_{e}\right)=\frac{\sigma_{i o n} \Gamma}{\arctan \left(\frac{E_{e}+E_{n}-E_{t h}}{2 \Gamma}\right)} .
$$

Since the energy-differential cross section is nomalized as

$$
\int_{0}^{E_{e l, m a}} \frac{\sigma_{i o n}\left(E_{e}, E_{e l}\right)}{\sigma_{i o n}} d E_{e 1}=1
$$

it iz possible to chooose a random number $R_{N}$ in the range $(0,1)$ to determine the kinetic energy of the scattered electron $E_{e 1}$, such that

$$
R_{N}=\int_{0}^{E_{e 1, \max }} \frac{\Gamma d E_{e 1}}{\left(E_{e 1}^{2}+\Gamma^{2}\right) \arctan \left(\frac{E_{e}+E_{n}-E_{l l}}{2 \Gamma}\right)}
$$

or rearranging

$$
E_{c 1}=\Gamma \tan \left(R_{N \arctan }\left(\frac{E_{c}+E_{n}-E_{t h}}{2 \Gamma}\right)\right) .
$$

The kinetic energy of the new electron and ion are tien determined by a mass-energy balance relatiun

$$
\begin{gathered}
E_{e 2}=\left(\Delta E-E_{e 1}\right)\left(\frac{m_{i}}{m_{i}+m_{c}}\right) \\
E_{i}=\left(\Delta E-E_{e 1}\right)\left(\frac{m_{e}}{m_{i}+m_{e}}\right),
\end{gathered}
$$

where $m_{e}$ and $m_{i}$ are the masses of the electron and lon respectively. Note that this method does not conserve hinetic energy, since the thireshold cuergy $\left(E_{t h}\right)$ is required to renove the bound electron from the atom.

The velocity componeuts ( $v_{\|}$and $v_{1}$ ) of the scattered electron, and the new chertron and ion are all chosen randomly

$$
\begin{aligned}
& v_{\|}=\sqrt{\frac{2 E}{m}} \cos \left(\pi R_{N}\right) \\
& v_{\perp}=\sqrt{\frac{2 E}{m}} \sin \left(\pi R_{N}\right)
\end{aligned}
$$


where $R_{N}$ is a random number in the range $(0,1)$. This implies that the incident electron velocity is scat tered isotropically during the ionizing collision. These velocity components are then used to calculate the parallel momentum $p_{z}$ and magnetic moment $\mu$ of the new electron and ion. The spatial location of the new electron/ion pair is assumed to be the same as that of the incident electron. By placing the new electron and ion at the same position, there is no net contribution to the net cliarge density $\rho(z)$ and the electric field $\mathbf{E}(z)$ arising from the ionization cvent.

\subsubsection{The Charge Exchange Model}

A charge exchange rvent occurs when a neutral atom transfers one or more electrons to an adjacent ion. This process results in a change in the kinetir energy of the cliarged and neutral species, with the ion and neutral particle densities reinaining constant. Charge exchange is the most probable interaction between ions and fusion-relevant neutral particles [14]. Therefore, it is the only ion/neutral collision process simulated by the code DIPSI.

The algorithm for the charge exchange model is similar to that described alrove for the impact ionization model. The probability of the $k$-th plasma ion exchanging at least one electron with a neutral particle during a given time step is also a function of the local neutral density $n_{n}(z)$ (defined on the grid points), the jon kinetic energy and velocity $E_{i} \equiv \frac{1}{2} m_{i}\left(v_{\| i}^{2}+v_{L_{i}}^{2}\right)$ and the time step duration $\Delta t$

$$
P_{c x, k}=\left[1-\exp \left(-n_{n}(z) \sigma_{c x}\left(E_{i, k}\right) v_{i, k} \Delta t\right)\right]
$$

The probability $P_{c x, k}$ is calculated for each ion in a given grid cell. A charge exchange event occurs if a random number $R_{N}$ in $(0,1)$ is less than $P_{c F, k}$. (As above, if the argument of the exponential in (24) is very small compared to unity, (i. e. $P_{c r, k} \ll 1$, it is passible to sample every $N_{\text {stip }}$ ions, and multiply the right side of (24) by $N_{\text {stip }}$ to provide a reasoltable probability per sampled ion per time step.)

The charge-exchange cross section for hydrogenic particles has been fit to measured dala by Riviere $[17,14]$. This empirical fit has the following dependence on the ion kinetic energs

$$
\sigma_{c x}\left(E_{i}\right)=\hat{\sigma}_{c x}\left(\frac{\left[1-0.155 \log \left(E_{i}\right)\right]^{2}}{1+1.112 \times 10^{-15} E_{i}^{3.3}}\right)
$$

where $\hat{\sigma}_{e s}$ is the maximun charge-exchange cross section, and $E_{i}$ is the ion kinetir energy in nuits of eV. 'This fit is ased in the prosent model. Therefore, the encrgy dejondence of 
the cross section is assumed to be the sanc for various ion/nentrad particle combinitions, but the magnitude may be changed via the input parameter $\hat{\sigma}_{c x}$. The energy dependesice of the charge-exchange cross section for protons intaracting with monatomic hydroge $\left\{{ }_{1} \|{ }^{1}\right\rangle$ is shown in Figure 6. Figure 6a shows experimentadly measured values [18], while Figure $6 \mathrm{~b}$ presents the values obtaited from (25) for $\hat{\sigma}_{c x}=0.94 \times 10^{-15} \mathrm{~cm}^{2}$. The empirical fit (25) provides excellent agreement with measured values over the energy range $0.1 \mathrm{eV} \lesssim E_{\mathrm{i}} \lesssim$ $1.0 \mathrm{MeV}$ (even though Figure 6h only covers the range $0.1 \mathrm{eV} \leq E_{i} \leq 2.0 \mathrm{keV}$ ).

The velocity components $v_{\perp}$ and $v_{\|}$of the ion are modified as a result of the chisge exchange event. However, the positions of the initial and final ions are the sane. The velocity components of the new ion ate dependent upon the temperature of the neutral atoms $k T_{n}$. This thermal spread is assumed to be isotropic and Maxwellian in Cartesian velocity space, such that the speed of the new ion is given by

$$
v_{i}^{\prime}=\left[\left(2 k T_{n} / m_{n}\right) \ln \left(\frac{1+\delta}{1+\delta-\Omega_{N}}\right)\right]^{1 / 2}
$$

where $\delta=10^{-8}, \Omega_{N}$ is a random number in $(0,1)$ and $m_{n}=m_{i}+Z m_{e}$ is the mass of the neutral atom of atomic number $Z$. The same metlod was used to deterinine the kinetic energy of the neutral particle in the inpact ionization model described above. The velocity components are then chosen randomly by $v_{\|, i}^{\prime}=v_{i}^{\prime} \cos \left(\pi R_{N}\right)$ and $v_{\perp, i}^{\prime}=v_{i}^{\prime} \sin \left(\pi R_{N}\right)$, where $R_{N}$ is a random number in the range $(0,1)$. These velocity components are then used to calculate the parallel momentum $p_{z}$ and magnetic moment $\mu$ of the new ion.

The impact jonjzation and charge excliange models have been used in [7] to study the interaction of cold, recycled, neutral gas and warm piasma, and its effect on the trausport of particles and energy througl the scrape-ofl layer to the divertor plate in a tokanak. 


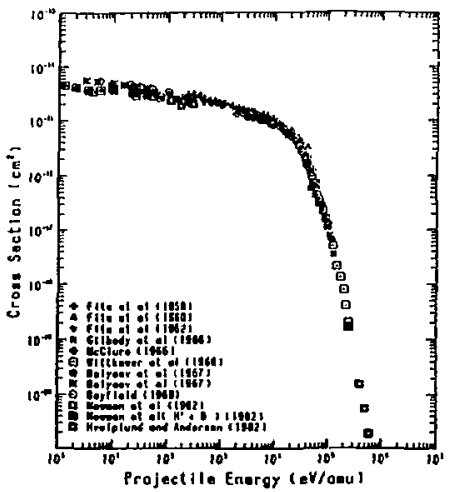

(a) Experimentally measured charge-exchange cros6 section [14].

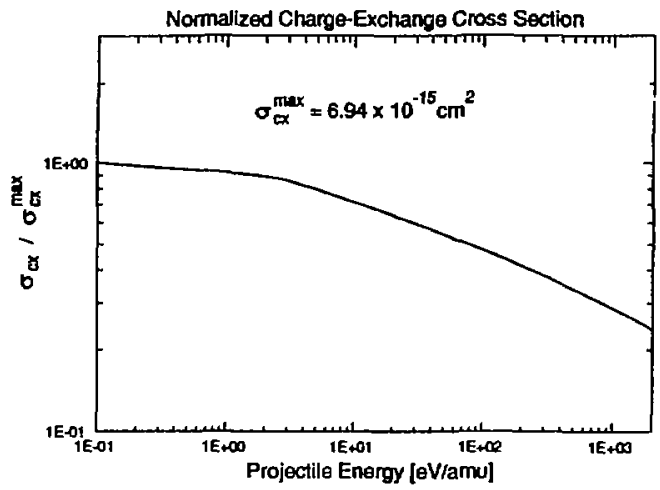

(b) Charge-exchange cross section calculated by the empirical fit (4.25).

Figure 6: Charge-exchange crose section as a function of energy for protons ( $\left.1 p^{1}\right)$ interacting with monatomic hydrogen $\left({ }_{1} H^{1}\right)$ from (a) experimental measurements [14] and (1.) from the empirical fit (4.25). 


\section{Acknowledgments}

The author would like to acknowledge V. Vahedi, M. V. Alves, I. J. Morcy and M. Surendra (University of California at Berkeley) for helpful discussions on the inodeling of charged/neutral interactions in a plasma. The code DIPSI is a version of the particle-ilt-celi code TESS, which was developed jointly by B. I. Cohen, R. J. Procassini and M. E. Stewart to stuldy confinement and transport in mirror plasmas. This research was supported $b_{y}$ a grant from the Plasma Physics Researcli Institute of the Lawrence Livermore Nationa' Laboratory. Additional funding was provided to the Plasma Theory and Simulation group at the University of California at Berkeley by the U. S. Department of Energy under Contract Number DE-FG03-86ER53220.

\section{References}

[1] R. J. Procassini and B. I. Cohen, "The DIPSI Conlputer Code User's Manlual", Report UCRL-ID 104093, Lawrence Livermore National Laboratory, Livermore (1990).

[2] W. S. Lawson, "PDW1 User's Manual", Memo UCB/ERL M84/37, Electronies Research Laboratory, University of California, Berkeley (1984).

[3] L. A. Schwager and C. K. Birdsall, Phys. Fluids B 2, 1057 (1930).

[4] C. K. Birdsal? and A. B. Langdon, Plasina Physics via Compuler Simulation, McGrawIIill, New York, pp. 393-394 (1985).

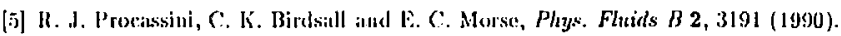

[6] R. J. Procassini and C. K. Birdsall, "Particle Simulation Model Of Transport In $A$ Bounded, Coulomb Collisiunal Plasma", submitted for publication in Phys. Fluids B, May 1990.

[7] R. J. Procassini, C. K. Birdsall and B. I. Colien, Nucl. Fusion 30, 2329 (1990).

[8] ITEIt Mallagement Coinmitlee, International Thermonuclear Experimental Reactor (ITER) Concept Definition, Report ITER-1, International Atomic Energy Agency, Vienna, Sec. II.3.2.5, VI.1.2 and X.2(1988). 
[9] W. Eckstein and H. Verbeek, in Data Compendium for Plasma-Surfact Intcrurlions, (Nucl. Fusion, Special Issue 1984), pp. 12-27 (1984).

[10] K. L. Witson, in Dala Compendium for Plasma-Surface Inteructions, (Nuct. Fusion, Special Issue 1984), pp. 28-42 (1984).

[11] E. Tagtaucr, in Data Compendium for Plasma-Surface Interactions, (Nucl. Fusion, Special Issue 1984), pp. 43-54 (1984).

[12] I. J. Morey, V. Vahedi and J. Verboncoeur, Bull. Am. Phys, Sor. 34, 2028, P'aper IU 15 (1989).

[13] V. Valedi, I. J. Morcy and M. V. Alves, private communication.

[14] R. K. Janev, W. D. Langer, K. Evans, Jr. and D. E. Post, "Atomic and Molecular" Processes in Iydrogen-Helium Plasmas", Report PPPL-TM-368, Princeton Plasma Pliysics Laboratory, 'rinceton (1985).

[15] H. Tawara, T. Kato and M. Onishi, "Ionization Cross Sections Of Atons and lons By Electron Impact", Report IPPJ-AM-37, Institute of Plasma Physics, Nagoya University, Nagoya (1985).

[16] L. R. Peterson and J. E. Allen, Jr., J. Chem. Phys. 56, 6068 (1972).

[17] A. C. Riviere, Nucl.Fusion 11, 363 (1971).

[18] 11. Tawara, T. Kato and Y. Nakai, "Cross Sections lor Charge Transfers Of Iliglily Ionized Ions In Hydrogen Atoms", Report 1PPJ-AM-30, Institute of Plasına P'hysics, Nagoya University, Nagoya (1983). 